\title{
Underwater Backscatter Networking
}

\author{
Junsu Jang and Fadel Adib \\ MIT Media Lab \\ \{junsuj,fadel\}@mit.edu
}

\begin{abstract}
We present Piezo-Acoustic Backscatter (PAB), the first technology that enables backscatter networking in underwater environments. $\mathrm{PAB}$ relies on the piezoelectric effect to enable underwater communication and sensing at near-zero power. Its architecture is inspired by radio backscatter which works well in air but cannot work well underwater due to the exponential attenuation of radio signals in water.

PAB nodes harvest energy from underwater acoustic signals using piezoelectric interfaces and communicate by modulating the piezoelectric impedance. Our design introduces innovations that enable concurrent multiple access through circuit-based frequency tuning of backscatter modulation and a MAC that exploits the properties of PAB nodes to deliver higher network throughput and decode network collisions.

We built a prototype of our design using custom-designed, mechanically fabricated transducers and an end-to-end battery-free hardware implementation. We tested our nodes in large experimental water tanks at the MIT Sea Grant. Our results demonstrate singlelink throughputs up to $3 \mathrm{kbps}$ and power-up ranges up to $10 \mathrm{~m}$. Finally, we show how our design can be used to measure acidity, temperature, and pressure. Looking ahead, the system can be used in ocean exploration, marine life sensing, and underwater climate change monitoring.
\end{abstract}

\section{CCS CONCEPTS}

- Networks $\rightarrow$ Network architectures; • Hardware $\rightarrow$ Wireless integrated network sensors; • Applied computing $\rightarrow$ Environmental sciences;

\section{KEYWORDS}

Subsea IoT, Piezoelectricity, Backscatter Communication, Wireless, Energy Harvesting, Battery-free

\section{ACM Reference Format:}

Junsu Jang and Fadel Adib. 2019. Underwater Backscatter Networking . In SIGCOMM '19: 2019 Conference of the ACM Special Interest Group on Data Communication, August 19-23, 2019, Beijing, China. ACM, New York, NY, USA, 13 pages. https://doi.org/10.1145/3341302.3342091

Permission to make digital or hard copies of all or part of this work for personal or classroom use is granted without fee provided that copies are not made or distributed for profit or commercial advantage and that copies bear this notice and the full citation on the first page. Copyrights for components of this work owned by others than ACM must be honored. Abstracting with credit is permitted. To copy otherwise, or republish, to post on servers or to redistribute to lists, requires prior specific permission and/or a fee. Request permissions from permissions@acm.org.

SIGCOMM '19, August 19-23, 2019, Beijing, China

(C) 2019 Association for Computing Machinery.

ACM ISBN 978-1-4503-5956-6/19/08 . .\$15.00

https://doi.org/10.1145/3341302.3342091

\section{INTRODUCTION}

Backscatter is the lowest power wireless communication technology, which has led to its widespread adoption for ultra-low power networking [1, 43, 48, 56, 87]. Backscatter sensors can wirelessly communicate at near-zero power by simply reflecting radio signals in the environment. In this paper, we investigate the ability to take backscatter networking to underwater environments. In particular, since wireless communication is the largest source of energy consumption for many underwater sensors [61, 81], transitioning to backscatter technology would eliminate the need for batteries which increase size and cost and require frequent replacement [41]. Batteryfree underwater sensors would enable us to sense ocean conditions (such as acidity, temperature, and bacteria content) over extended periods of time and understand how they correlate with climate change [44]. Scientists may attach these sensors to marine animals and use them to understand migration and habitat patterns [77]. Such sensors may even be used in space missions to search for life in the recently discovered subsurface oceans of Saturn's moon, Titan [52]. More generally, underwater battery-free sensors can be leveraged in many long-term ocean applications such as naval deployments, oil spill monitoring, and scientific exploration.

Unfortunately, existing backscatter networks are intrinsically incapable of operating underwater. This is because they rely on radio signals, which die exponentially in water [26, 38], making them undesirable for underwater communication and power harvesting. In contrast, underwater communication typically relies on acoustic signals, which can travel over long distances underwater [67, 68]. Indeed, that is why submarines rely on acoustic signals (e.g., SONAR) rather than radio signals for communication and sensing [79].

To enable underwater backscatter networking, we exploit the piezoelectric effect. ${ }^{1}$ This effect refers to the ability of certain materials to generate electrical energy in response to an applied mechanical stress [64]. Since acoustic signals travel as pressure waves, they would induce a strain (deformation) on a piezoelectric material, causing it to transform the pressure wave into a voltage; hence, this effect has been used in designing certain kinds of microphones [27]. More importantly, the piezoelectric effect is reversible, meaning that electrical signals applied on the electrodes of a piezoelectric device can be used to generate acoustic signals. It is this reversibility that makes piezoelectricity an enabler for underwater backscatter communication.

We introduce Piezo-Acoustic Backscatter (PAB), a system that enables underwater networking at near-zero power. We explain PAB's high-level operation through an analogy to radio backscatter in Fig. 1. In radio frequency $(\mathrm{RF})$ backscatter, a transmitting antenna sends a signal on the downlink, and an RF backscatter node harvests energy from this signal and communicates by modulating its reflection. On

\footnotetext{
${ }^{1}$ Note that the term "backscatter" is often used in the underwater literature to refer to SONAR-based imaging of objects by reflection $[15,22]$ rather than for communication or networking, which is the goal of this paper.
} 


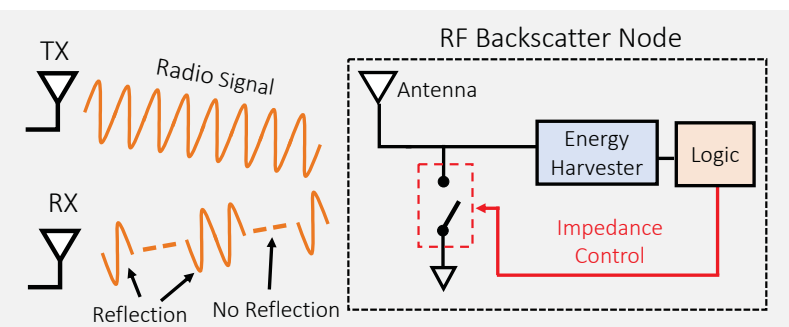

(a) RF Backscatter in Air

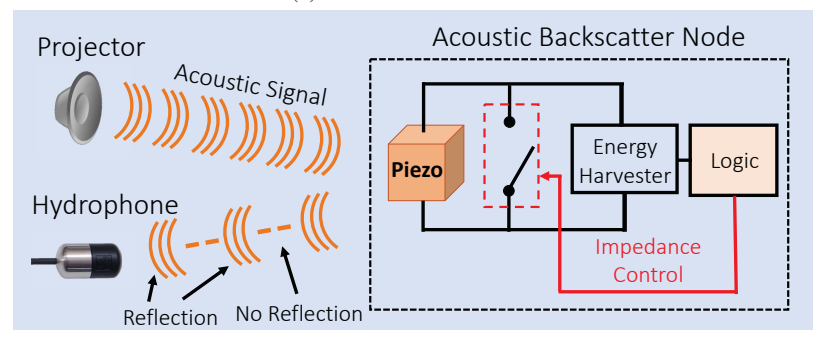

(b) Piezo-Acoustic Backscatter in Water

Figure 1-Analogy between RF and Piezo-Acoustic Backscatter. (a) shows how radio backscatter can communicate bits of zero and one by controlling the antenna impedance switch. (b) shows how PAB system communicates bits of zero and one by controlling the piezoelectric impedance switch. Note that in the absorptive states, the sensor can harvest energy.

the other hand, a PAB system consists of an acoustic projector (transmitter), a hydrophone (receiver), and a battery-free sensor. When the projector transmits acoustic signals underwater, a PAB sensor harvests energy from these signals and communicates by modulating their reflections. In particular, it can transmit a ' 0 ' bit by absorbing the incoming energy, and a ' 1 ' bit by reflecting the impinging acoustic signal. It can switch between the reflective and absorptive states by modulating the voltage across the piezoelectric interface, which in turn determines its vibration amplitude (i.e., reflection). The hydrophone receives the acoustic signals, senses changes in the amplitude due to reflection, and decodes these changes to recover the transmitted messages.

Interestingly, $\mathrm{PAB}$ operates a piezoelectric material as a reflector by preventing it from deforming (i.e., nulling the strain). Intuitively, if the material cannot deform, it is unable to absorb the incoming acoustic signal and must reflect it entirely. To do so, the node can simply activate a switch that connects the device's electrodes as shown in Fig. 1(b). Such switching requires near-zero power and can be done entirely using the harvested energy, enabling PAB sensors to be battery-free. In $\S 3$, we describe this process in detail and the underlying physics of piezo-acoustic backscatter.

A fundamental challenge facing PAB networks, however, is that piezoelectric materials are typically designed to operate at a specific resonant frequency [8]. ${ }^{2}$ While operating at resonance improves their sensitivity and range of operation, it also limits their bandwidth. This prevents different links from concurrently communicating over multiple channels as in standard WiFi or cellular networks. Said differently, it precludes the adoption of medium access control (MAC) protocols like frequency-division multiple access (FDMA), where concurrent links occupy different parts of the frequency spectrum.

\footnotetext{
${ }^{2}$ This is typically denoted by a high quality factor 'Q' (quality factor) [76], which is the ratio of the resonant frequency to the bandwidth.
}

To overcome this challenge and enable multiple PAB sensors to transmit concurrently, our idea is to shift the piezoelectric resonance frequency itself across the different sensors. If different sensors have slightly different resonance frequencies, then they would occupy different bands of the acoustic frequency spectrum, naturally leading to FDMA. Hence, if different projectors transmit acoustic signals at different frequencies, each would activate a different sensor operating at the corresponding resonance frequency, thus enabling concurrent multiple access. The hydrophone receives all the reflected signals and applies software-based filters in order to isolate and decode the colliding backscatter reflections.

To realize this idea, we introduce the concept of recto-piezos. Recto-piezos are acoustic backscatter nodes whose resonance frequency can be tuned through programmable circuit matching. The design of recto-piezos is inspired by a concept from RF-based energy harvesting called rectennas [46]. Rectennas can optimize their energy harvesting efficiency by matching the impedance of the rectifier (energy harvester) to the antenna. This impedance matching results in a resonance mode, at which rectennas are known to optimally harvest energy. While recto-piezos are inspired by this idea, they instead employ it for tuning their resonance frequency. This matching-based frequency tuning allows us to ensure that different sensors have different resonance frequencies, and enable multiple concurrent backscatter transmissions, thus improving the network throughput.

We built a prototype of PAB's design and tested it in large experimental water tanks at the MIT Sea Grant. Each battery-free node consists of a mechanically fabricated piezoelectric resonator, potted in polyurethane for acoustic matching, and a custom-made PCB that incorporates the recto-piezo, the energy harvesting unit, a microcontroller that implements the backscatter logic, and a general and extensible peripheral interface that can be integrated with different sensors.

Our results demonstrate that PAB sensors achieve communication throughputs up to $3 \mathrm{kbps}$ and power-up ranges up to $10 \mathrm{~m}$. We also demonstrate how our recto-piezo design enables tuning the resonance frequency and shifting it to an adjacent channel. This enables doubling the network throughput through concurrent transmissions and collision decoding.

To show the potential of our design, we implement proof-ofconcepts for three sensing tasks. PAB nodes are integrated with sensing interfaces that can measure acidity $(\mathrm{pH})$, temperature, and pressure. Such sensors may be powered by the node's harvested energy and the microcontroller samples their analog output or communicates with their digital interface using one of its peripherals. Our evaluation demonstrates the ability to correctly sense these measurement conditions, enabling long-term ocean condition monitoring. It is worth noting that while these applications can work well with modest throughputs, PAB's throughput could support more demanding applications such as recording sound or low-quality images of marine animals and plants.

Contributions: PAB is the first underwater backscatter communication system. It introduces a new backscatter technology and sensor architecture that exploits the piezoelectric effect for backscatter networking. It also introduces recto-piezo, a programmable resonance design that enables multiple PAB sensors to operate concurrently. 
The paper also contributes a prototype implementation and evaluation of a battery-free platform for ocean sensing and communication.

Our current design still exhibits limitations in its modest throughput and range. These limitations are primarily imposed by the downlink signal and the desire to keep the implementation battery-free through energy harvesting. In principle, one could achieve higher throughputs and ranges by adapting battery-assisted backscatter implementations from RF designs [59], which would enable deep-sea deployments and exploration, while still inheriting PAB's benefits of ultra-low power backscatter communication. The design and implementation of such hybrid systems is outside the scope of this paper and left for future work. More generally, we hope that PAB's approach for underwater networking would follow a similar trend in throughput and range improvements witnessed by radio backscatter in recent years.

\section{BACKGROUND}

The piezoelectric effect was discovered in 1880 by the Curie brothers [49]. They observed that certain types of crystals generate an electric charge when they undergo a mechanical strain (deformation). The following year, they demonstrated that this process is reversible, and an electric signal applied on piezoelectric crystals induces a strain. Since its discovery, this phenomenon has been widely used in various applications including Quartz clocks, buzzers, inkjet printers, and X-ray shutters [13].

Our work directly builds on two main areas of piezoelectric research in the context of underwater environments: energy harvesting and acoustic transducers. In particular, since the piezoelectric effect can transform mechanical energy to electrical energy, it has been used to harvest energy from different kinds of underwater movements, including those resulting from swimmer body movements [20], fish movements [14, 40], water currents [70, 78], motor vibrations [39], and even ambient noise [65]. Moreover, due to their high electromechanical conversion efficiency, piezoelectric resonators have been used in designing a wide array of underwater transducers [12]. PAB's design builds on this past work for energy harvesting and electromechanical translation. Our contributions are orthogonal and focus on exploiting the piezoelectric effect to enable underwater backscatter communication and developing protocols for underwater backscatter networking.

It is worth noting that the term backscatter is widely used in the context of underwater sensing $[22,29,60]$. The usage of the term refers to SONAR-based imaging, similar to how radar imaging is often called backscatter $[18,32]$. This is different from the networking community's use of the backscatter term to refer to communication by modulating reflections $[43,48,82]$, which is the focus of this work.

Finally, our work advances the recently growing interest in batteryless underwater communication $[24,40]$. In contrast to PAB, all existing systems communicate by generating their own acoustic carriers, which requires multiple orders of magnitude more energy than backscatter communication [85]. As a result, existing systems need to harvest power for long periods of time (e.g., from fish movements [40]) before they have enough energy to generate an acoustic beacon. As a result, their average throughput is limited to few to tens of bits per second $[31,40]$. PAB shares the same motivation of this line of work and pushes its boundaries by introducing underwater backscatter, which significantly decreases the energy required for transmissions and boosts the network throughput by two to three orders of magnitude.

\section{PIEZO-ACOUSTIC BACKSCATTER}

In this section, we first describe the basic physical principles that enable backscattering acoustic signals in underwater environments, then describe how PAB uses these principles to design an underwater backscatter networking system.

\subsection{Piezoelectric Transducers}

Before we explain piezo-acoustic backscatter, we describe how the piezoelectric effect is typically employed for underwater acoustic communication. A piezoelectric transducer can transform acoustic signals into electrical signals at the same frequency, and vice versa [12]. In order to transmit acoustic signals, we can apply a voltage on a piezoelectric device, causing it to vibrate at the same frequency of the applied voltage. The vibration creates a pressure wave which travels as an acoustic or ultrasonic signal depending on the vibration frequency.

For simplicity, assume that we would like to transmit a sine wave at a single frequency $f$. If we apply a signal with some peak voltage $V$ to the piezoelectric device, that results in the following transmitted pressure wave:

$$
P=\alpha V \sin (2 \pi f t+\phi)
$$

where $t$ is time, $\phi$ is the phase offset, $\alpha$ is a proportionality constant which depends on various factors including the type of piezoelectric material, transducer geometry, and frequency of operation [12]. Naturally, while the above discussion focuses on a single sine wave, it can be extended to any modulation scheme (BPSK, QAM, OFDM, etc.) by multiplying the sine wave by the desired baseband signal as in typical wireless communication [80].

\subsection{Backscattering Acoustic Signals}

In standard underwater acoustic communication, a transmitter generates and amplifies the carrier signal (i.e., the sine wave), a process that consumes most of the transmitter's energy. Even low-power acoustic transmitters typically require few hundred Watts [67, 83]. Below, we show how a PAB sensor can employ backscatter to communicate without generating any carrier wave, which enables it to communicate at near-zero power.

Recall that backscatter communication involves switching between reflective and non-reflective states. In the non-reflective (absorptive) state, a PAB sensor can simply operate in a manner similar to a standard piezoelectric receiver (hydrophone), transforming a pressure wave into an electric signal. However, the reflective state is not straightforward and hence is the focus of our discussion.

To demonstrate that it is possible to transform a piezoelectric material into a reflector, we resort to the fundamental physical process that governs the converse piezoelectric effect. Piezoelectric materials respond to both electrical and mechanical stimuli. Said differently, an electric field (E) or a tensor/stress (T) applied on the material causes charge accumulation (D) at its terminals. We can express the 
relationship using the following equation [12]:

$$
\underbrace{D}_{\text {charge displacement }}=\underbrace{d T}_{\text {mechanical }}+\underbrace{\epsilon^{T} E}_{\text {electrical }}
$$

where $d$ is the piezoelectric coefficient, and $\epsilon^{T}$ the permittivity coefficient under constant stress. The above equation shows the coupled nature of piezoelectric materials.

In order to backscatter an incoming acoustic signal, PAB turns on a switch that connects the two terminals of the piezoelectric device as shown in Fig. 1(b). Doing so ensures that the charge distribution $D$ and the electric field $E$ are both set to zero in the steady state (since there is no voltage or charge when the terminals are shorted). Substituting these values into Eq. 1 demonstrates that the net tensor $T$ experienced by the piezoelectric material must be zero.

But how can the tensor (stress) be zero in the presence of an incoming acoustic signal (from the projector) which induces pressure on the piezoelectric material? To answer this question, observe that the above relationship depends on the net tensor experienced by the piezoelectric material. We can express the net tensor as the sum of the incoming pressure wave from the projector $\left(P_{i n}\right)$ and the reflected pressure wave $P_{\text {ref }}$. Hence, when the two terminals of a piezoelectric device are connected, any incoming signal will be entirely reflected as per the following equation:

$$
P_{\text {ref }}=T-P_{\text {in }}=-P_{0} \sin (2 \pi f t)
$$

where $P_{0}$ is the amplitude of the incident pressure wave caused by the acoustic signal. This derivation demonstrates the power of backscatter, whereby the backscattering node does not need to generate its own carrier but rather relies on an external one for communication.

We note that our above discussion is simplified in two ways. First, the derivation assumes that the piezoelectric device itself is lossless. In practice, some of the incoming signal is dissipated due to heat and, as a result, the amplitude of the reflected wave is smaller than that of the incident wave. Second, while the net tensor is directly related to the applied pressure, this relationship may also involve a multiplicative factor which depends on the geometry and polarization of the piezoelectric material [12] as well as the angle of the incoming pressure wave with respect to the surface of the resonator.

Physical Interpretation. So far, by looking at the relationship governing the charge displacement $D$, we have demonstrated that a piezoelectric material can be used as a reflector. Next, we are interested in gaining insight into the mechanical interpretation of this process. Recall that a piezoelectric material deforms when a tensor (stress) or an electric field is applied to its terminals. The total deformation (strain) is given by the following equation:

$$
S=s^{E} T+d E
$$

where $s^{E}$ is the compliance coefficient under constant electric field. As per our above discussion, when the piezoelectric material is in reflective state, both the electric field and the net tensor are zero. This means that the total strain is also nulled. The mechanical interpretation demonstrates that PAB transforms the piezoelectric material into a reflector by preventing it from deforming. This ensures that an incoming pressure wave must be entirely reflected, further demonstrating the reflective phenomenon from a mechanical perspective.

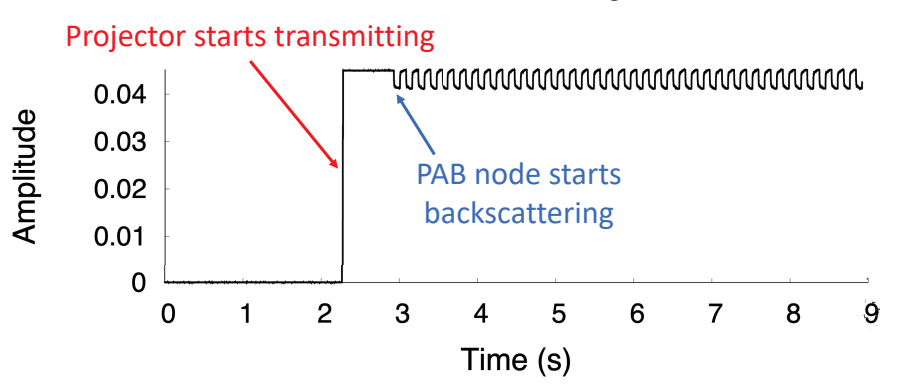

Figure 2-Received and Demodulated Backscatter Signal. The figure plots the received signal after demodulation and low-pass filtering. After PAB starts backscattering, the larger amplitude corresponds to the reflected state while the smaller amplitude corresponds to the non-reflected state. The node switches between these two states to communicate bits of $0 \mathrm{~s}$ and $1 \mathrm{~s}$ (using FM0 encoding).

Testing the Waters. We run an experiment with a PAB sensor underwater. We use an underwater projector to send an acoustic signal at $15 \mathrm{kHz}$ and configure the PAB sensor to backscatter the signals by switching its impedance between reflective and non-reflective every $100 \mathrm{~ms}$. The design of the sensor is detailed in $\$ 4$. We receive the signal using a hydrophone and demodulate by removing the carrier frequency.

Fig. 2 plots the amplitude of the received output over time. The plot exhibits a jump to a constant amplitude at $t=2.2 \mathrm{~s}$, which corresponds to the start of the downlink transmission from the projector. Note that even though the projector transmits at $15 \mathrm{kHz}$, we only see a constant amplitude since downconversion removes the carrier wave and only keeps its amplitude. At $t=2.8 \mathrm{~s}$, the amplitude of the received signal starts alternating between two states. This corresponds to the time when the PAB node starts backscattering and the two amplitudes correspond to the two reflective states. The change in amplitude between the node's two states is weaker than the amplitude of the constant wave transmitted by the projector. This is due to two reasons: first, the signal from the backscatter node travels a longer distance than the one coming directly from the projector (since it travels from the projector to the node and back to the hydrophone) and hence experiences larger attenuation. Second, the backscatter process is lossy, meaning that the reflected signal will be weaker than the incident one. These observations are in line with those of radio backscatter [82], and the experiment validates PAB's ability to communicate via piezo-acoustic backscatter.

Now that we have demonstrated underwater backscatter, our design adapts few additional components from RF backscatter designs [23, 85]:

- Modulation: Our above discussion focused on demonstrating the ability to switch between two reflective states to enable backscatter, and abstracted the two states as bits of ' 0 ' and ' 1 '. In practice, backscatter communication can be made more robust by adopting modulation schemes like FM0 or Manchester encoding [82], where the reflection state switches at every bit, enabling the receiver to better delineate the bits and robustly decode backscatter signals. Hence, PAB adopts FM0 modulation on the uplink.

- Energy Harvesting \& Battery-Free Operation: The absorptive state of backscatter modulation provides an opportunity to harvest energy from the projector's downlink communication signal since it involves a conversion between mechanical and electrical energy. Similar to passive RFIDs, PAB harnesses this opportunity. It employs a rectifier and capacitor to store this energy, and uses 
it for controlling the backscatter switch. This energy may also be used for powering various onboard sensors. Moreover, to ensure maximum power transfer and optimize energy harvesting in the absorptive state, our front-end employs an impedance matching network (to match the load impedance $Z_{L}$ to the conjugate of the piezoelectric source $Z_{S}$ ). We also adopt the Pulse Width Modulation (PWM) scheme on the downlink since it can be decoded using simple envelope detection, thus minimizing power consumption during backscatter and since it provides ample opportunities for energy harvesting [23].

- Maximizing the Signal-to-Noise Ratio (SNR): In order to maximize the (SNR) and hence the throughput, we would like to maximize the difference between the reflected power in the two reflective states of backscatter communication. To do so, we rely on the standard backscatter reflection equation $[37,53]$ :

$$
P_{r}=\left|\frac{Z_{L}-Z_{s}^{*}}{Z_{L}+Z_{s}}\right|^{2} P_{i}
$$

where $P_{r}$ and $P_{i}$ are reflected and incident power respectively, and $*$ denotes the complex conjugate. Recall that in the reflective state, the two terminals are shorted (i.e., $Z_{L}=0$ ), and hence the entire incident wave is reflected. Thus, to maximize the SNR, we need to minimize the reflected power in the absorptive state. This can be achieved by setting $Z_{L}=Z_{s}^{*}$. Notice that this is the same impedance that maximizes the energy transfer as discussed above.

\subsection{From Communication to Networking}

A fundamental challenge facing PAB networks is that piezoelectric materials are designed to resonate at specific frequencies [12]. ${ }^{3}$ On one hand, operating at resonance maximizes the energy transfer between mechanical and electrical states, which improves both the sensitivity and the energy harvesting efficiency. On the other hand, it limits the operation bandwidth of piezoelectric energy harvesters. The limited bandwidth prevents adopting MAC protocols like FDMA where different backscatter nodes may transmit concurrently, each occupying its own frequency channel.

In this section, we describe how PAB overcomes this challenge through a hardware-based resonance tuning design and a receiver design that can decode concurrent transmissions.

\subsubsection{Recto-Piezos}

To better understand the problem of limited bandwidth of PAB's piezoelectric sensors, we run an experiment where we measure the voltage at the output of the rectifier of a backscatter node. This voltage is directly related to the harvested energy and used to power up the node. The backscatter node used in this experiment is optimized to harvest energy around $15 \mathrm{kHz}$ (as per the impedance matching process described in §3.2). We transmit a continuous wave signal at $15 \mathrm{kHz}$ using our projector, and measure the received voltage at the output of the rectifier. We repeat the experiment multiple times, each time transmitting at a different center frequency.

Fig. 3 plots the rectified voltage as a function of frequency using a solid black line. The plot shows that the rectified voltage (and hence the harvested energy) reaches its maximum of $4 \mathrm{~V}$ around the resonant frequency of $15 \mathrm{kHz}$. However, the voltage drops rapidly as

\footnotetext{
${ }^{3}$ This is typically referred to as having a high quality factor $Q=\mathrm{f} /$ Bandwidth [76].
}

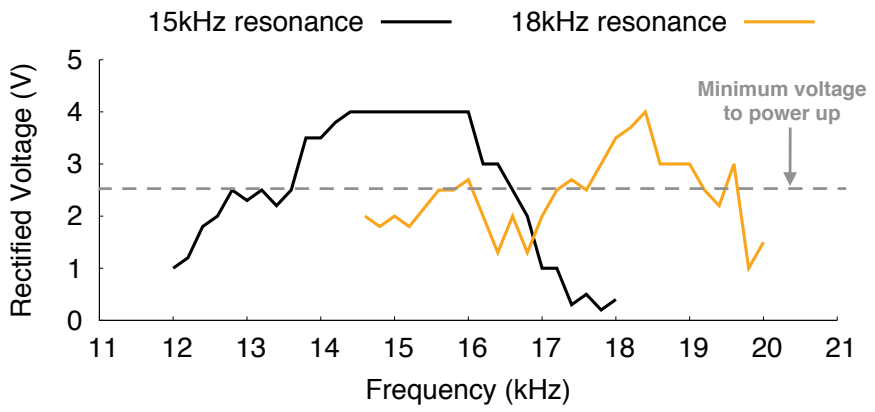

Figure 3-Rectopiezo. The figure plots the rectified voltage as a function of the downlink transmit frequency for two different recto-piezo configurations. One of the rectopiezos (in black) is electrically matched at $15 \mathrm{kHz}$ and the second (in orange) at $18 \mathrm{kHz}$.

we move away from that frequency. When the downlink frequency is outside $13.6-16.4 \mathrm{kHz}$, the voltage drops below $2.5 \mathrm{~V}$, which is the minimum required by our backscatter node to consistently power up for sensing and communication.

To overcome this bandwidth constraint and enable concurrent transmissions at multiple different frequency channels (i.e., via FDMA), ${ }^{4}$ PAB shifts the resonance frequency itself. In particular, recall from Eq. 2 that backscatter operates by alternating between reflective and absorptive states, and that these states can be realized by properly matching the load impedance, $Z_{L}$, to the source impedance, $Z_{s}$. To shift the resonance frequency, PAB exploits the fact that the impedance $Z(f)$ itself is a function of frequency. Indeed, this is why the voltage falls off in Fig. 3 beyond a certain bandwidth. This frequency dependence provides an opportunity to enable concurrent transmissions, each of which is matched to a different resonance. Said differently, we can design different sensors with matching circuits that are optimized to different center frequencies. We call this design recto-piezo as it exploits the matching properties of the piezoelectric interface to the rectifier to tune the resonance frequency. 5

To test this idea, we design another backscatter node, this time with a recto-piezo optimized to resonate at $18 \mathrm{kHz}$. The projector sends a downlink signal at $18 \mathrm{kHz}$, and we plot the resulting rectified voltage as a function of frequency in orange in Fig. 3. The plot also shows that the rectified voltage rises above the threshold around the new resonance frequency and over a bandwidth of $1.5 \mathrm{kHz}$. Also, notice that the responses of the two recto-piezos are complementary. This suggests that these sensors can operate concurrently while occupying different frequency channels, thus increasing the overall network throughput.

\subsubsection{Decoding Collisions}

In standard FDMA systems, when two transmitters occupy different channels, a receiver may apply a filter around the center frequency of interest in order to isolate the desired transmission and eliminate interference from the concurrent transmission on another channel. Unfortunately, this does not hold true for backscatter transmissions.

\footnotetext{
${ }^{4}$ Note that CDMA requires the same overall bandwidth as standard FDMA since it uses a spreading code at a higher rate than the transmitted signals, thus requiring a larger frequency (as it is a spread spectrum technology).

${ }^{5}$ It is important to note that while the recto-piezo can shift the resonance frequency through impedance matching, the overall efficiency decreases well outside the geometric resonance of the piezoelectric material itself. Said differently, the geometric resonance acts as a bandpass filter, while the electrical matching determines the exact frequency.
} 
The difficulty in isolating transmissions from different frequencies arises from the fact that backscatter modulation is frequencyagnostic [48]. Specifically, a backscatter node communicates by reflecting incoming signals. Hence, as long as a node powers up (due to a downlink signal within its resonance bandwidth), it would backscatter and modulate the reflections of all signals impinging on it, even those outside its resonance frequency. ${ }^{6}$ As a result, even if a recto-piezo requires a downlink signal at a resonance frequency of $18 \mathrm{kHz}$ to power up, it would also backscatter signals at $15 \mathrm{kHz}$, thus interfering with a concurrent transmission at the $15 \mathrm{kHz}$ range. Indeed, when we tried operating both recto-piezos from \$3.3.1 concurrently, the hydrophone received colliding signals and was unable to decode them.

To overcome this challenge, we observe that the two backscatter transmissions not only collide at $15 \mathrm{kHz}$ but also at $18 \mathrm{kHz}$. This provides the hydrophone receiver with two equations and two unknowns. Moreover, because the matching process results in frequency selectivity, the wireless channel experienced by the backscattered signals would be different. Mathematically, the hydrophone receiver would obtain the following two signals:

$$
\begin{aligned}
& y\left(f_{1}\right)=h_{1}\left(f_{1}\right) x_{1}+h_{2}\left(f_{1}\right) x_{2} \\
& y\left(f_{2}\right)=h_{1}\left(f_{2}\right) x_{1}+h_{2}\left(f_{2}\right) x_{2}
\end{aligned}
$$

where $y$ is the received signal, $f_{1}$ and $f_{2}$ are the resonance frequencies, $x_{1}$ and $x_{2}$ are the backscattered signals by the two the recto-piezos, and $h_{1}$ and $h_{2}$ are their corresponding frequencyselective channels.

The above behavior is similar to the one obtained in MIMO communication between two-antenna nodes. MIMO exploits spatial diversity, while our decoding scheme exploits frequency diversity. ${ }^{7} \mathrm{By}$ performing standard channel estimation, we can invert the channel matrix and decode the two signals using standard MIMO decoding techniques [80].

Two additional points are worth noting:

- Our above discussion focused on the physical and MAC layers of the communication system. Our design extends to encompass the entire networking stack and incorporates a protocol similar to that adopted by RFIDs. Specifically, the projector is similar to an RFID reader and transmits a query on the downlink which contains a preamble, destination address, and payload. Similarly, the uplink backscatter packet consists of a preamble, a header, and a payload which includes reading from on-board sensors as we describe in $\$ 5.1$.

- Our description of recto-piezo assumes that each backscatter node can operate at a single predefined resonance frequency. This design may be easily extended through programmable hardware to enable the backscatter node to shift its own resonance frequency. This may be achieved by incorporating multiple matching circuits onboard the backscatter node and enabling the micro-controller to select the recto-piezo.

\footnotetext{
${ }^{6}$ The modulation depth of backscattered signals (i.e., difference between reflected and absorbed power) decreases as their frequency moves away from resonance due to the degradation in impedance matching and efficiency.

${ }^{7}$ We note that our system is not the first to propose decoding backscatter collisions [82]; however, by exploiting the concept of recto-piezo, our proposed approach makes the decoding matrix better conditioned, which improves diversity.
}

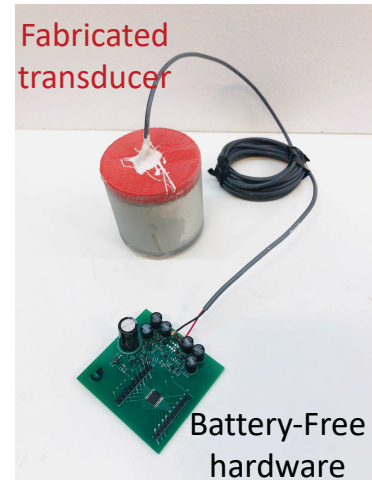

(a) PAB node

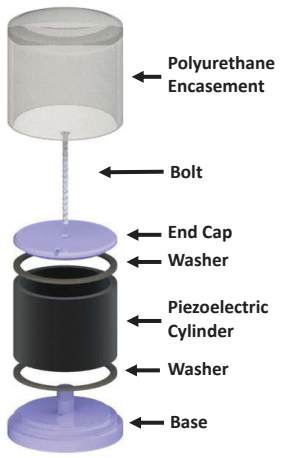

(b) Exploded transducer view
Figure 4-Mechanical and Hardware Fabrication. (a) shows the mechanically fabricated transducer with the battery-free analog-digital hardware design, (b) shows the exploded transducer view.

\section{MECHANICAL FABRICATION AND HARDWARE DESIGN}

In this section, we describe our mechanical and hardware fabrication process for PAB's backscatter nodes.

\subsection{Transducer Fabrication}

Fig. 4(a) shows a photo of one of our fabricated transducers. The main component of the transducer is a piezoelectric cylinder. We purchased a ceramic cylinder from Stemnic with an in-air resonance frequency of $17 \mathrm{kHz}$ [66], a radius of $2.5 \mathrm{~cm}$, and a length of $4 \mathrm{~cm}$. The cylinder vibrates radially making it omnidirectional in the horizontal plane. The choice of in-air resonance of $17 \mathrm{kHz}$ was intended to create balance between the size of the transducer, the propagation characteristics of acoustic signals underwater, and throughput. Specifically, lower acoustic frequencies experience less attenuation in underwater environments [11], but they also have narrower bandwidths (which further limits their throughput) and require larger cylinder dimensions since the dimensions of the resonator are inversely proportional to its frequency [12]. ${ }^{8}$ Nonetheless, we note that our design is general and can be adapted to different applications that have different range, size, and throughput requirements.

Fig. 4(b) shows an exploded view of our transducer design. Our design follows prior proposals for low-cost underwater transducers and is adapted to the geometry of our cylinder and its operation frequency $[6,28] .{ }^{9}$ We encapsulated the piezoelectric resonator with a polymer and added end-caps on the top and bottom of the cylinder. The encapsulation and end-caps serve to insulate the electrodes from water (preventing it from shorting the electrodes) and to prevent water from flowing inside the cylinder and loading its resonance. Such design is called an air-backed, end-capped transducer and is known to provide high efficiency in electromechanical conversion [12].

\footnotetext{
${ }^{8}$ For example, a resonator with center frequency of $500 \mathrm{~Hz}$ can propagate over $1000 \mathrm{~km}$, but has a bitrate of $100 \mathrm{bps}$ and is $3600 \times$ larger than our cylinder [51].

${ }^{9}$ There are various design choices for an underwater piezoelectric transducer; the efficiency and directionality of each design depend on various parameters including the type of piezoelectric material, shape of the transducer, backing and encapsulants used, etc. To demonstrate proof-of-concept of underwater backscatter, we followed state-of-the-art transducer prototypes from the literature.
} 
To build the transducer, we first soldered two wires to the two electrodes of the piezoelectric ceramic (i.e., the inner and outer surfaces of the cylinder). We 3D printed the base and the top cap from Fig. 4 as well as a cylindrical mold to house the ceramic cylinder and encapsulation polymer. We placed polyurethane washers [50] on the top and bottom of the cylinder, then placed it on a base and added a top cap. The washers enable the cylinder to vibrate freely without being loaded by the end-caps. The setup is held tight using a screw and locking nut, then placed inside the mold.

To prepare the encapsulation polymer, we used the polyurethane WC-575A mixture from BJB enterprises [9]; this polymer was chosen due to its transparency and because its acoustic impedance maximizes the energy transfer between water and the piezoelectric material. We prepared the polyurethane by mixing its components as per the manufacturer's guidelines [9] then placed the resulting mixture inside a vacuum tank to extract any moisture or residual air bubbles from the mixture. We poured the prepared polyurethane into the cylindrical mold and left it to pot for 12 hours in a pressure chamber at $60 \mathrm{psi}$ (4atm). Once the potting was done, we removed the mold and added marine epoxy [45] to seal any remaining holes and ensure that water does not leak into the transducer. We fabricated eight of these transducers in total.

As part of our fabrication process, we also experimented with fully-potted (i.e., non-air-backed) designs, but noticed that these designed had poorer sensitivity and energy harvesting efficiency than air-backed transducers. This observation is shared by past research [28]. We also experimented with various encapsulation thicknesses but did not observe a significant difference in their performance.

\subsection{Hardware Design \& Fabrication}

Our hardware design is entirely battery-free and was designed and fabricated on a two-layer printed circuit board (PCB). The design serves four primary purposes: backscatter communication (for uplink), energy harvesting, receiving and decoding (for downlink signals), and interfacing with peripherals. This architecture enables $\mathrm{PAB}$ to serve as a general-purpose and extensible platform for battery-free underwater sensing and communication.

The overall schematic of our designs is shown in Fig. 5. We used Autodesk Eagle design software [5] to design the schematic and layout. A total of 20 boards were manufactured by PCBWay [55] at the cost of $\$ 10$. The circuit components were hand-soldered on the PCBs and individually tested.

\subsubsection{Mixed Analog-Digital Circuit Design}

A piezoelectric resonator provides a differential output (rather than a single-ended output with a ground). Hence, our analog front-end adopts a differential design as can be seen in the mirrored architecture in Fig. 5, where the upper and lower portion of the energy harvesting and backscatter units are mirror images of each other. ${ }^{10}$

Backscatter. To enable backscatter communication, we insert two transistors in series between the two terminals of the piezoelectric device. The middle junction of the two transistors is connected to ground, enabling symmetric backscatter and maximizing the SNR

\footnotetext{
${ }^{10}$ Not accounting for the differential design could lead to unstable performance since the digital components of the design need to be grounded.
}

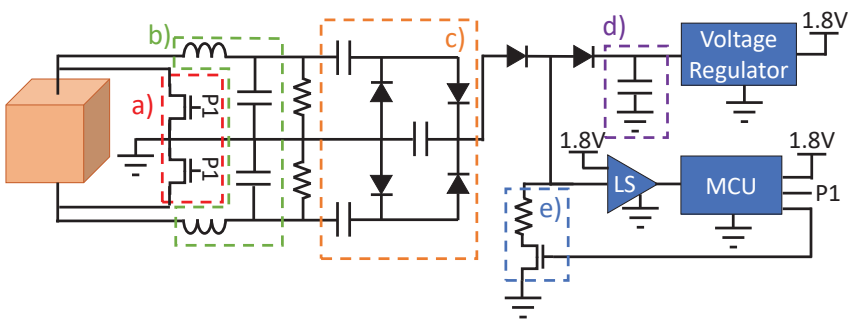

Figure 5-Representative Circuit Schematic of PAB. The figure shows the circuit schematic of PAB with a differential analog front-end. The components are: (a) Switches that control the load impedance to enable backscatter communication, (b) Matching network, (c) Rectifying circuit that converts AC to DC voltage and passively amplifies the voltage, (d) Supercapacitor that stores energy from the rectified DC voltage, (e) Pull-down transistor for improving the SNR of the downlink signal.

of the backscattered signal. ${ }^{11}$ The transistors act as switches to enable toggling the piezoelectric interface between reflective and non-reflective stages, when they are operating in short-circuit and open-circuit modes, respectively. The gates of the transistors are controlled by the microcontroller described in $\$ 4.2 .2$.

Energy Harvesting. To harvest energy from acoustic signals, we use a rectifier followed by a storage capacitor as in standard energy harvesting designs [47]. The rectifier transforms the alternating electrical signal coming from the transducer into a DC voltage by passing it through diodes and capacitors. We employ a multi-stage rectifier in order to passively amplify the voltage to the level that is needed for activating the digital components of the circuit design. The rectified DC charge is stored in a $1000 \mu \mathrm{F}$ supercapacitor.

Recall from $\S 3$ that maximizing the energy harvesting efficiency requires matching the output impedance of our piezoelectric transducer to the input impedance of the load $\left(Z_{L}=Z_{s}^{*}\right)$. We use the E4990A Impedance analyzer [35] to measure the output impedance of the transducer as well as the input impedance of the load. Knowing these impedances, we can solder an impedance matching network (which consists of an inductor and a capacitor) between the piezoelectric transducer and the rectifier. The values of inductance and capacitance of the network can be derived from standard circuit equations by substituting the load and source impedances [54].

The capacitor is connected to a low-dropout (LDO) voltage regulator, the LP5900SD [74], the output of which is $1.8 \mathrm{~V}$. The voltage regulator drives the digital components of the circuit, ensuring they are not damaged or operated in an unsteady mode.

Decoding. PAB's decoder operates by envelope detection. In particular, recall that the downlink communication signal is encoded using PWM, where a larger pulse width corresponds to a ' 1 ' bit and a shorter pulse width corresponds to a ' 0 ' bit. In order to decode these pulses, the receiver needs a simple edge detection mechanism to identify the bit (pulse) boundaries and durations.

Our receiver design is inspired by the RFID WISP platform [85]. The design employs a Schmitt trigger that can discard small amplitude changes due to noise and discretize the output into two main voltage levels: high and low. A level shifter scales the voltage levels to properly condition them as inputs to a microcontroller. We use the TXB0302 [72], which incorporates both a trigger and a level shifter.

\footnotetext{
${ }^{11}$ This series design enables controlling the switches at a lower gate-to-source voltage $\left(V_{G S}\right)$ since the source is always at ground. This allows the microcontroller to switch between the two states at a lower threshold voltage.
} 
Our design also employs a pull-down transistor, as shown in Fig. 5 to improve both the energy harvesting efficiency and the decodability of the downlink signal. Specifically, the pull-down transistor acts as an open-circuit in the cold-start phase (i.e., when the super-capacitor is charging) to ensure that all the incoming energy flows to the capacitor enabling fast charging. Once the capacitor has enough voltage to power on the voltage regulator and the microcontroller (MCU), the MCU applies a voltage on the pull-down transistor changing it to a closed circuit. While this leaks some of the energy to ground, it also maximizes the difference between the high and low voltage levels at the input to the Schmitt trigger, thus improving the SNR for decoding the downlink PWM signal.

\subsubsection{Microcontroller}

To minimize the power consumption of our design, we use an ultralow power microcontroller, specifically the MSPG2553 MCU[75]. The microcontroller can operate with a supply voltage as low as $1.8 \mathrm{~V}$ and consumes less than $230 \mu \mathrm{A}$ at $1.8 \mathrm{~V}$ in active mode and $0.5 \mu \mathrm{A}$ in low power mode (LMP3) with just one active clock using a crystal oscillator operating at $32.8 \mathrm{kHz}$.

Upon powering up, the MCU prepares to receive and decode a downlink command by enabling interrupts and initializing a timer to detect a falling edge at its pin which is connected to the output of the level shifter; then, it enters LMP3 mode. A falling edge at the microcontroller's input raises an interrupt waking up the MCU, which enters active mode to compute the time interval between every edge to decode bit ' 0 ' or ' 1 ' of the query, before going back to low-power mode.

Upon successfully decoding downlink signals from the projector, the MCU prepares for backscatter. It switches the timer to continuous mode to enable controlling the switch at the backscatter frequency and employs FM0 encoding as described in $\$ 3$. An output pin of the microcontroller is connected to the two switching transistors enabling them to toggle the transducer between reflective and nonreflective states.

The microcontroller can also be configured to communicate with analog and digital peripheral sensors. The ADC pin is used for sampling analog sensors and the $I^{2} C$ protocol is used to communicate with digital sensors.

\section{EVALUATION}

\subsection{Implementation}

Our implementation setup consists of an acoustic projector, PAB backscatter nodes, and a hydrophone receiver. The backscatter nodes are designed and implemented as per our discussion in $\S 4$, so we describe the remaining system components below.

(a) Transmitter. We implemented the transmitter using our in-house transducers from $\S 4.1$ as projectors. Each projector was connected to the output audio jack of a Lenovo Thinkstation PC through the XLi2500 Two-channel 750W power amplifier [16]. We configure the projector to transmit signals at different center frequencies between $12 \mathrm{kHz}$ and $18 \mathrm{kHz}$. For different configurations, we change the matching circuit to optimize the power transfer between the power amplifier and the transducer. The transmitted signal is generated using MATLAB and employs PWM where the ' 1 ' bit is twice as long as the ' 0 ' bit. The transmitter's downlink query includes a 9-bit

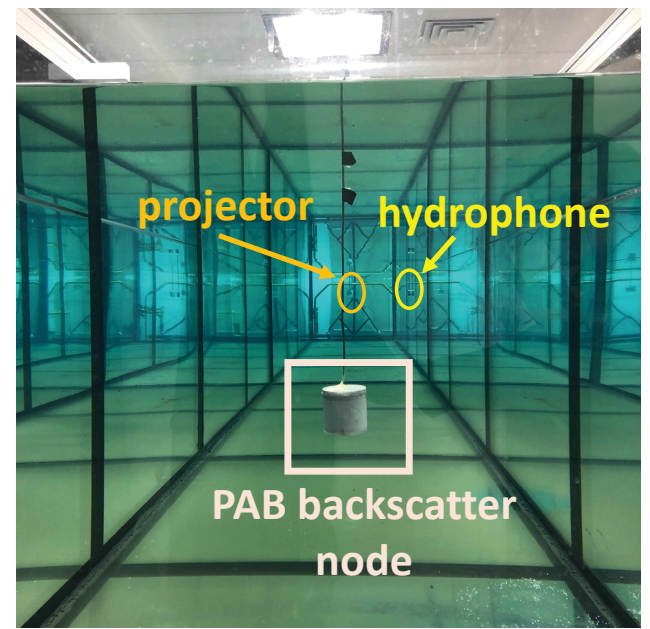

Figure 6-Evaluation Setup. The figure shows one of the evaluation setups in the long tank (Pool B) with a projector, hydrophone, and a PAB sensor.

preamble. The transmitter packet may also include commands for the PAB backscatter node such as setting backscatter link frequency, switching its resonance mode, or requesting certain sensed data like $\mathrm{pH}$, temperature, or pressure.

(b) Receiver. The receiver is implemented using the H2a hydrophone [3], whose sensitivity is $-180 \mathrm{~dB}$ re: $1 \mathrm{~V} / \mathrm{A} t ̧ \mathrm{~Pa}$. The hydrophone is connected to the audio jack of a Lenovo Thinkstation. We use the Audacity software package to record the received audio signals. The signals are processed offline using a MATLAB-based decoder. The decoder identifies the different transmitted frequencies on the downlink using FFT and peak detection. It then downconverts the signals to baseband by multiplying each of them with its respective carrier frequency. The receiver then employs a Butterworth filter on each of the receive channels to isolate the signal of interest and reduce interference from concurrent transmissions. Subsequently, it performs standard packet detection and carrier frequency offset (CFO) correction using the preamble. ${ }^{12}$ It then employs a maximum likelihood decoder to decode the FM0 decoded bits. It can also use the CRC to perform a checksum on the received packets and request retransmissions of corrupted packets.

(c) Sensing Applications. We tested three types of sensing applications by interfacing the micro-controller with different sensors. To measured acidity, we use the Mini pH Probe [4], which connects to micro-controllers ADC using an LMP91200 Configurable Analog Front End for Chemical Sensing Application [73]. We also experimented with the MS5837-30BA Waterproof Digital Pressure Sensor [71] to extract temperature and pressure. The sensor has a digital interface which directly communicates with the MCU through $I^{2} \mathrm{C}$ communication protocol.

(d) Setups. We evaluated PAB in two main settings: Pool A is an enclosed water tank of $1.3 m$ depth and $3 m \times 4 m$ rectangular crosssection, and Pool B is another enclosed water tank of $1 \mathrm{~m}$ depth and $1.2 m \times 10 m$ rectangular cross section. We also validated that the

\footnotetext{
${ }^{12}$ In contrast to RF backscatter where the reader is typically full-duplex, PAB uses a separate transmitter (projector) and receiver (hydrophone). Hence, the receiver observes a CFO due to the different oscillators.
} 


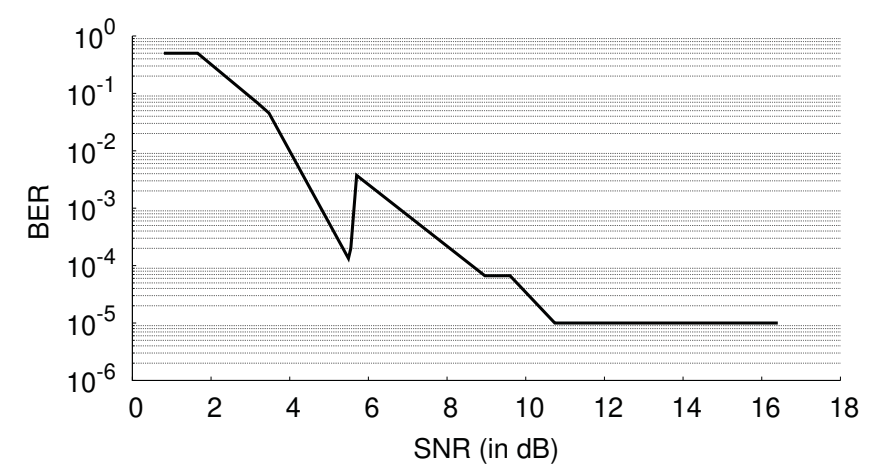

Figure 7-BER-SNR Curve. The figure plots the BER of the decoded backscatter signal as a function of the SNR of the signal received by the hydrophone.

system operates correctly in an indoor swimming pool. Throughout our evaluation, the transducers were submerged between $50 \mathrm{~cm}$ and $1 \mathrm{~m}$ below the surface, and the distance between the projector, hydrophone, and backscatter nodes were varied across our experimental trials.

\section{RESULTS}

To evaluate $\mathrm{PAB}$, we performed controlled experiments in the enclosed water tanks described in $\$ 5.1$. Our experiments test the ability to use PAB for communication and study the communication and energy harvesting performance as a function of distance, backscatter rate, and concurrent transmissions.

We performed over 150 experimental trials in total. We varied the backscatter rate, location, and depth of PAB's transducers throughput these experiments. We also varied the distance and placement with respect to the projector and hydrophone.

\subsection{Communication Performance}

(a) BER-SNR Curves. First, we are interested in testing the ability to communicate underwater using piezo-acoustic backscatter. The performance of a communication system can be evaluated through plots of the bit error rate to the signal to noise ratio, called the BER-SNR curves $[25,58]$. We computed the BER as the fraction of correctly decoded bits to the total transmitted bits. We computed the signal power as the squared channel estimate, and computed the noise power as the squared difference between the received signal and the transmitted signal multiplied by the channel estimate.

Fig. 7 plots the BER-SNR curves of PAB in log-log scale. The plot is generated from our experimental trials across the various bitrates. As expected the BER decreases with increasing SNR. Our decoder is able to decode with a minimum SNR around $2 \mathrm{~dB}$, which is typical for biphase modulation techniques like FM0. The BER drops to $10^{-5}$ at SNRs higher than $11 \mathrm{~dB}$. We set the lowest BER to $10^{-5}$ since our packet size is always smaller than $10^{5}$ bits.

(b) Throughput Evaluation. Next, we are interested in evaluating the SNR as a function of backscatter bitrate. In particular, since higher backscatter bitrates occupy a larger bandwidth (while piezoelectrics have a limited bandwidth), we would like to understand the impact of bitrate on SNR. We place a battery-free PAB node in a fixed position, within a meter of both the projector and the

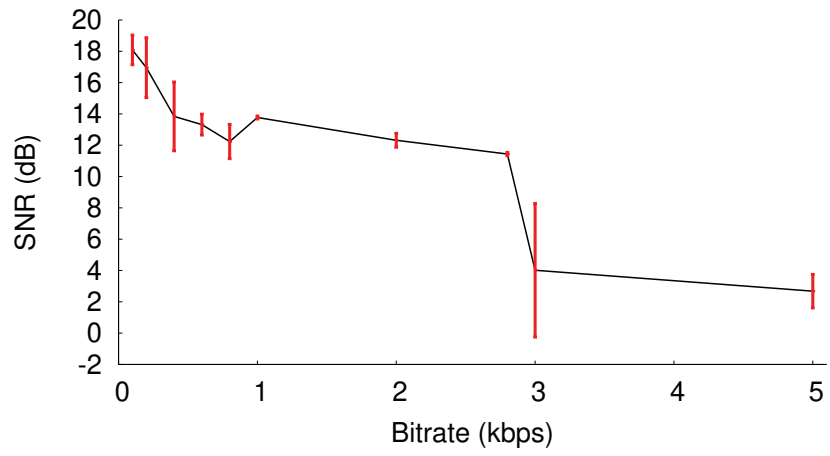

Figure 8-SNR vs Backscatter Bitrate. The figure plots the SNR of the signal received at the hydrophone when the PAB sensor is configured to backscatter at different bitrates. Error bars indicate standard deviation.

hydrophone. In each experimental trial, we configure its microcontroller to backscatter at one of the following bitrates: $100 \mathrm{bps}, 200 \mathrm{bps}$, 400bps, 600bps, $800 \mathrm{bps}, 1 \mathrm{kbps}, 2 \mathrm{kbps}, 2.8 \mathrm{kbps}, 3 \mathrm{kbps}$, and $5 \mathrm{kbps}$. We vary the bitrate at the backscatter node by configuring a different MCU clock divider, ${ }^{13}$ and repeat the same experiment three times for each bitrate. We compute the SNR the same as above.

Fig. 8 plots the average SNR and its standard deviation as a function of the backscatter bitrate. At the same distance, the SNR decreases when the bitrate increases. This is because a higher bitrate requires spreading the transmit power over a wider bandwidth. It is worth noting that the SNR significantly drops for bitrates higher than $3 \mathrm{kbps}$, which would result in very high bit error rates. This is partly because the efficiency of the recto-piezo reduces as the frequency moves from its resonance.

\subsection{Performance vs Distance}

Next, we would like to evaluate the distance over which we can power up a battery-free PAB node. To perform this evaluation against different multipath environments, we test the powering up range in both pools. Recall that a PAB node harvests energy from the downlink acoustic signal transmitted by a projector. So, in this experiment, we fix the voltage (and hence the power) input of the projector and measure the maximum range over which it can power the PAB sensor. We repeat the experiment at different transmit voltages and in both pools. Note that we do not report beyond $5 \mathrm{~m}$ for Pool A and $10 \mathrm{~m}$ for Pool B since these correspond to the largest range possible in each of the respective pools.

Fig. 9 plots the maximum distance as a function of the input voltage to the transducer. The figure shows that in both environments, the distance increases with the increased input voltage. This can be explained by the fact that higher voltage leads to higher transmit power, enabling the battery-free node to harvest energy to power up even at a longer range. Interestingly, the same transmit power enables a longer powering up range in Pool B than in the Pool A. This is likely because Pool B is elongated and acts as a corridor, focusing the projector's signal directionally, rather than omnidirectionally as in Pool A.

\footnotetext{
${ }^{13}$ The resolution with which we can vary the bitrate depends on the integer clock divider available in the MCU.
} 


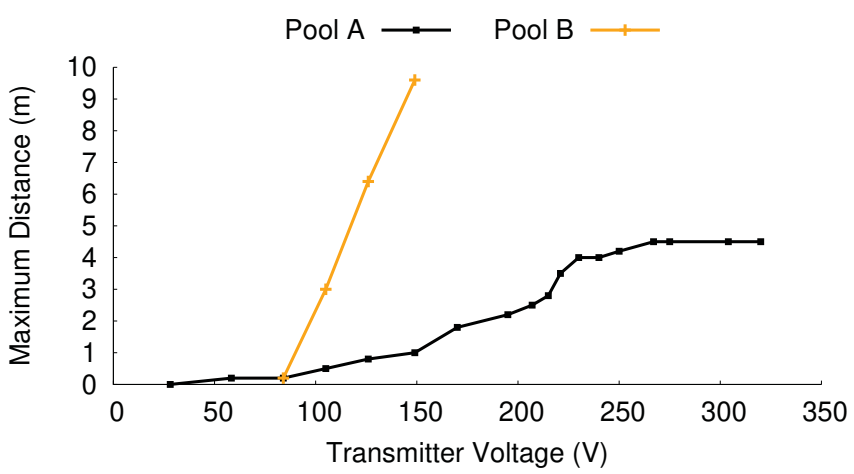

Figure 9-Maximum Distance vs Transmit Voltage. The figure plots the maximum distance at which the battery-free sensor node can be powered up remotely by the transmitter as the input voltage to the transmitter increases.

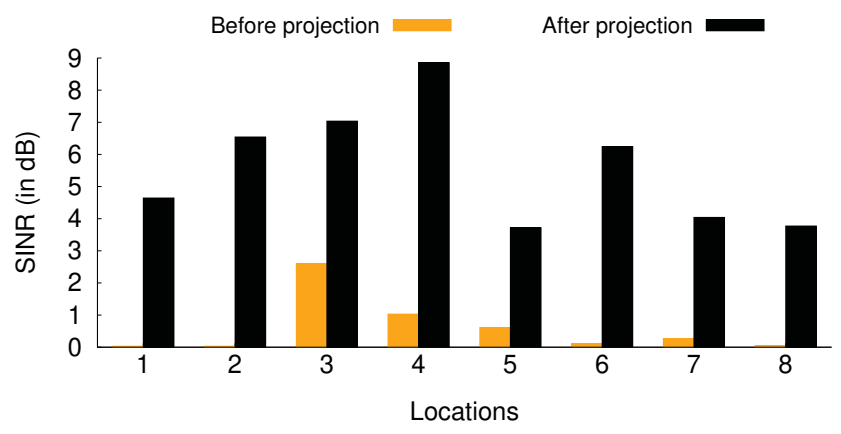

Figure 10 - System Performance with Concurrent Transmissions. The figure plots the SINR before (orange) and after (black) projection at 8 different locations.

\subsection{Concurrent Backscatter Transmissions}

Recall that PAB enables concurrent backscatter transmissions using the recto-piezo concept, where different nodes have different resonance frequencies and where the receiving hydrophone employs a MIMO-style decoder to deal with collisions. In order to test PAB's approach for enabling concurrent transmissions, we run experiments where we designed one battery-free sensor to resonate at $15 \mathrm{kHz}$ and another to resonate at $18 \mathrm{kHz}$. We create an audio file for the projector which transmits a downlink signal at both frequencies. We vary the location of the two backscatter nodes across experimental trials. The receiving hydrophone captures the concurrent backscatter transmissions, downconverts them using their respective resonance frequencies and applies MIMO channel inversion (i.e., it decodes by zero-forcing through projecting on the orthogonal of the unwanted channel vector [80]). We measure the SINR (signal-to-interferenceand-noise ratio) of each of the backscattered signals before and after projection.

Fig. 10 plots the SINR before and after projection across eight of the locations. We make the following remarks:

- The SINR before projection is less than $3 \mathrm{~dB}$ across all the trials. This low SINR is due to the fact that the concurrent backscatter transmission interferes at the receiver, even though it is programmed to resonate at a different frequency. Recall from $\$ 3.3 .2$ that this is due to the frequency-agnostic nature of backscatter. We note that if the projector only transmits at one frequency, then the SNR would be higher because the second recto-piezo would not power up altogether. However, this would defeat the purpose of enabling multiple concurrent transmissions via FDMA.

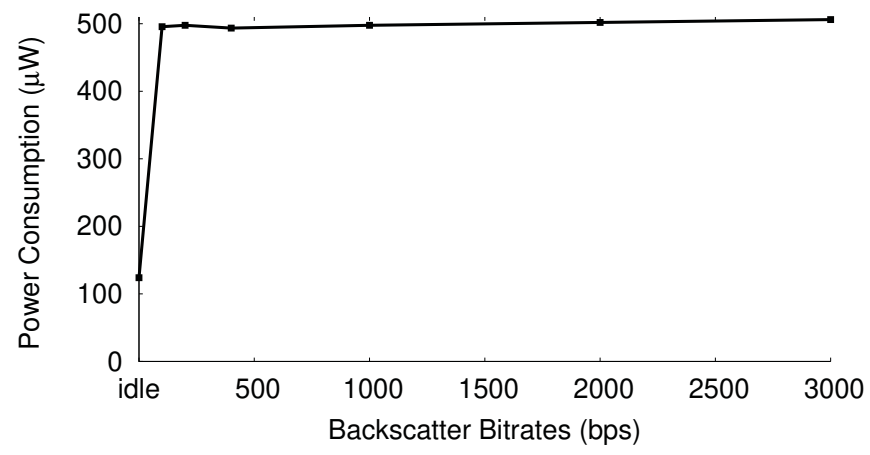

Figure 11- Power Consumption vs Backscatter Bitrate. The figure plots the overall power consumption of the system as it backscatters at different data rates. The system is idle when it is ready to receive and decode a downlink signal.

- Even though the SINR is relatively low before projection, it is still greater than zero. This is because even though the concurrent transmissions interfere, the backscatter node operating at resonance will have a stronger channel than the interfering one (which is backscattering out-of-band).

- After projecting and inverting the channel, the SINR is significantly boosted and is greater than $3 \mathrm{~dB}$ across the recorded locations, indicating the ability to decode the transmissions despite interference as per $\$ 6.1(\mathrm{a})$. Moreover, since the capacity of a wireless channel is directly proportional to the SINR, our results demonstrate that PAB's decoding approach can boost network throughput and enable concurrent multiple access.

- Finally, it is worth noting that the SINR after projection is different across locations. This is expected since the wireless channel changes with location.

\subsection{Power Consumption}

Next, we would like to evaluate the power consumption of a PAB sensor. We used the Keithley 2400 source meter [34] to measure the current draw, and computed the power as the product of the current and the voltage when the sensor is connected to a power supply. It is important to note that in all our previous evaluation, the sensor was powered remotely and that connecting it to a power supply (instead of the supercapacitor) is only used in this section for accurately measuring power consumption.

Fig. 11 plots the power consumption when the sensor is in idle state (waiting to receive and decode a downlink signal) as well as when it is backscattering at $100 \mathrm{bps}, 200 \mathrm{bps}, 400 \mathrm{bps}, 1 \mathrm{kbps}, 2 \mathrm{kbps}$, and $3 \mathrm{kbps}$. The figure shows that the power consumption in idle state is $124 \mu \mathrm{W}$ and increases to around $500 \mu \mathrm{W}$ for the different backscatter bitrates. These power consumption numbers are in line with those expected from the datasheets of PAB's components. In particular, the microcontroller, which is the most power-consuming component of PAB's design, draws around $230 \mu \mathrm{A}$ in active mode (i.e., when backscattering), and the LDO draws around $25 \mu \mathrm{A}$ at the corresponding current draw from the MCU. These current measurements are obtained when the voltage into the LDO is $2.1 \mathrm{~V}$, making the product of total current draw and voltage within $7 \%$ of the datasheets specifications.

Interestingly, the measured power consumption in idle mode is higher than the datasheet specifications. We identify two contributing factors for this discrepancy. First, the MCU is not entirely in standby 
since it sets few pins to high (the pull-down transistor, interrupt handles). Second, the LDO consumes similar power even when the $\mathrm{MCU}$ is in standby. This suggests more room for reducing the power consumption.

\subsection{Applications}

Finally, we show how a PAB sensor can be used to measure $\mathrm{pH}$, temperature, and pressure. Having demonstrated the powering up and communication aspects of our design, we focus our evaluation on integrating sensing interfaces with the microcontroller and embedding data into transmitted packets.

pH: As described in $\$ 5.1(\mathrm{c}), \mathrm{pH}$ measurements are done using a miniprobe connected to the microcontroller's ADC via a signal conditioning circuit. The ADC samples the output voltage, transforms it to a numerical $\mathrm{pH}$, embeds it into the payload of a backscatter packet, and transmits it from a PAB sensor. We verified that the MCU computes the correct $\mathrm{pH}$ (of 7), thus demonstrating the correctness of our conversion and interfacing processes. We note that, in this integration, the signal conditioning circuit was powered using an external supply and used to amplify the probe's voltage measurements. Future iterations of the sensor design may eliminate the power supply by incorporating an onboard battery or by leveraging the harvested energy and duty-cycling the $\mathrm{pH}$ sensing process.

Temperature \& pressure: As described in §5.1(c), temperature and pressure measurements are done using a waterproof digital sensor which directly communicates with the MCU using an $I^{2} C$ interface. The digital sensor powers up by drawing current from the MCU. Similar to the $\mathrm{pH}$ measurements, we verified the correctness of this design through correct readings of room temperature and atmospheric pressure (around 1 bar).

The above evaluation demonstrates the extensibility of PAB's design, its ability to interface with various sensors, and its potential use for in-situ underwater measurements.

\section{RELATED WORK}

(a) RF backscatter \& Rectennas: RF Backscatter has gained significant attention from both the networking and the circuits communities over the past few years. Recent proposals have demonstrated the ability to backscatter and harvest energy from TV [43], WiFi [1], and LoRa [56] transmissions, all of which are radio signals and hence cannot work underwater. Circuits researchers have also introduced efficient energy harvesting designs called rectennas where the antenna and the rectifier are matched to improve sensitivity and energy harvesting efficiency [10, 86], as well as for tuning purposes [46]. $\mathrm{PAB}$ is inspired by literature in both communities and brings the benefits of backscatter communication to underwater environments.

(b) In-body Communication: Similar to underwater environments, the human body exponentially attenuates radio signals (since $60 \%$ of the body consists of water). This has led researchers to leverage ultrasonic piezoelectric materials for various in-body applications, including imaging [69], medical implant communication [21, 36], and neuronal sensing [62].

Recent systems have explored the use of piezoelectric materials for backscattering ultrasonic signals inside the human body [21, 36,
62]. PAB is similar to these past proposals in that it uses piezoelectricity to enable backscatter, but differs from them in its constraints, design, and capabilities. Specifically, PAB does not enjoy the wide bandwidth afforded by ultrasound piezoelectrics nor can it rely on highly directional scanners available in the ultrasound range. ${ }^{14}$ This precludes it from adopting the spatial or frequency diversity schemes employed by ultrasonic proposals which enable them to scale to multiple sensors. To address these challenges, PAB's design introduces multiple innovations including recto-piezos and a MAC protocol. Moreover, in terms of capabilities, because it relies on acoustic signals, PAB can operate over much longer distances than ultrasonic backscatter, making it a more attractive solution for underwater and ocean applications.

(c) Subsea IoT \& Underwater Sensor Networks: Finally, our work is related to a large body of research on subsea IoT and underwater sensor networks [33, 42, 79, 81], including DARPA's Ocean of Things initiative [17]. It is well-known that the battery life is one of the most important challenges facing this area of research $[33,42,63]$ and that wireless communication is the main source of battery drain for many applications [61, 81]. Most existing underwater communication systems rely on acoustic or optical transmissions $[30,68]$, which are known to be power hungry. To overcome this challenge, some proposals considered the use of magnetic induction for charging and communication [2, 19]. However, magnetic induction inherently suffers from a small distance-to-coil size ratio; hence, practical implementations of this approach have demonstrated a limited range of few millimeters to few centimeters $[7,57,84]$. PAB shares the motivation with this line of work and combines the long-range communication properties of acoustic signals with the ultra-low power nature of backscatter communication to enable practical ultra-low power underwater networking.

\section{DISCUSSION \& OPPORTUNITIES}

This paper presents $\mathrm{PAB}$, the first technology that enables underwater backscatter networking. ${ }^{15}$ The technology leverages the piezoelectric effect to enable battery-free underwater networking. As the research evolves, we envision it may be used in a wide variety of applications such as ocean exploration, marine life sensing, and underwater climate change monitoring. Below, we highlight potential development opportunities and discuss some of the challenges ahead:

- Operation Environment: As the research progresses, we would like to test and deploy the technology in more complex environments such as rivers, lakes, and oceans. While we expect the same principle to hold in such environments, the mechanically fabricated transducers need to be optimized for the corresponding environmental conditions, including temperature, pressure, and salinity. These settings are also likely to introduce new challenges, such as mobility and multipath, which would be interesting to explore.

- Transducer Tunability: Our design enables multiple access by tuning the resonance frequency of a PAB sensor. In principle, the

\footnotetext{
${ }^{14}$ These systems employ ultrasound signals whose wavelength is few hundreds of microns, i.e., $1000 \times$ larger than PAB's acoustic wavelength. The short wavelength enables building highly directional and compact ultrasonic scanners.

${ }^{15}$ This work does not raise any ethical issues.
} 
gain from FDMA scales as the number of nodes with different resonance frequencies increases. However, the tunability of a PAB sensor will be limited by the efficiency and bandwidth of the piezoelectric transducer design. This motivates novel transducer designs and MAC protocols to scale to a large number of nodes.

- Sensing Capabilities: Our evaluation demonstrates the ability to remotely power up the sensors and achieve communication throughputs of few kbps. The desire to increase range and data rates motivates further research to reduce power consumption and increase sensitivity, throughput, and deep-sea sensing capabilities

Acknowledgments. We thank Ali Fayed and Vivian Huang for help with the transducer fabrication and sensor integration. We also thank Canan Dagdeviren, Matthew Johnson-Roberson, and Eduardo Iscan for helpful discussions on piezoelectricity and transducer design. We also thank Thomas Consi for his help at the MIT Sea Grant. Finally, we thank our shepherd Kyle Jamieson, the anonymous SIGCOMM reviewers, and the Signal Kinetics group for their helpful feedback on the manuscript. This research is supported by the Office of Naval Research Young Investigator Award and the MIT Media Lab.

\section{REFERENCES}

[1] Ali Abedi, Mohammad Hossein Mazaheri, Omid Abari, and Tim Brecht. 2018 WiTAG: Rethinking Backscatter Communication for WiFi Networks. In Proceed ings of the 17th ACM Workshop on Hot Topics in Networks. ACM, 148-154.

[2] Ian F Akyildiz, Pu Wang, and Zhi Sun. 2015. Realizing underwater communication through magnetic induction. IEEE Communications Magazine 53, 11 (2015), 42 48.

[3] Aquarian Audio. 2019. H2a Hydrophone. http://www.aquarianaudio.com/ h2a-hydrophone.html. (2019).

[4] Atlas Scientific LLC. 2019. Mini pH Probe. https://www.atlas-scientific.com/ product_pages/probes/mini_ph_probe.html. (2019).

[5] Autodesk Inc. 2019. Eagle. https://www.autodesk.com/products/eagle/overview. (2019).

[6] B. Benson, Y. Li, B. Faunce, K. Domond, D. Kimball, C. Schurgers, and R. Kastner. 2010. Design of a Low-Cost Underwater Acoustic Modem. IEEE Embedded Systems Letters 2, 3 (Sep. 2010), 58-61. https://doi.org/10.1109/LES. 2010.2050191

[7] N. W. Bergmann, J. Juergens, L. Hou, Y. Wang, and J. Trevathan. 2013. Wireless underwater power and data transfer. In 38th Annual IEEE Conference on Local Computer Networks - Workshops. 104-107. https://doi.org/10.1109/LCNW.2013. 6758505

[8] S. Bhuyan, K. Sivanand, S. K. Panda, R. Kumar, and J. Hu. 2011. ResonanceBased Wireless Energizing of Piezoelectric Components. IEEE Magnetics Letters 2 (2011), 6000204-6000204. https://doi.org/10.1109/LMAG.2011.2167133

[9] BJB Enterprise. 2015. Water Clear Shore 70 A Polyurethane Elastomer. https: //bjbenterprises.com/index.php/wc-575-a-b/. (2015).

[10] W. C. Brown. 1984. The History of Power Transmission by Radio Waves. IEEE Transactions on Microwave Theory and Techniques 32, 9 (Sep. 1984), 1230-1242. https://doi.org/10.1109/TMTT.1984.1132833

[11] David Browning and R H. Mellen. 1987. Attenuation of Low-Frequency Sound in the Sea: Recent Results. 403-410. https://doi.org/10.1007/978-1-4613-1871-2_46

[12] J.L. Butler and Sherman C.H. 2016. Transducers and Arrays for Underwater Sound. Springer.

[13] Walter Guyton Cady. 2018. Piezoelectricity: Volume Two. Courier Dover Publications.

[14] Youngsu Cha, Woojin Chae, Hubert Kim, Horace Walcott, Sean D Peterson, and Maurizio Porfiri. 2016. Energy harvesting from a piezoelectric biomimetic fish tail. Renewable Energy 86 (2016), 449-458.

[15] James Churnside, Richard Marchbanks, Chad Lembke, and Jordon Beckler. 2017. Optical Backscattering Measured by Airborne Lidar and Underwater Glider. Remote Sensing 9 (04 2017), 379. https://doi.org/10.3390/rs 9040379

[16] Crown International. 2019. XLi 1500: Two-channel, 450W, 4Ohm Power Amplifier. (2019).

[17] DARPA. 2017. Ocean of Things Aims to Expand Maritime Awareness across Open Seas. https://www.darpa.mil/news-events/2017-12-06. (2017).

[18] Myron Craig Dobson, Fawwaz T Ulaby, Thuy LeToan, Andre Beaudoin, Eric S Kasischke, and Norm Christensen. 1992. Dependence of radar backscatter on coniferous forest biomass. IEEE Transactions on Geoscience and remote Sensing 30, 2 (1992), 412-415.
[19] Mari Carmen Domingo. 2012. Magnetic induction for underwater wireless communication networks. IEEE Transactions on Antennas and Propagation 60, 6 (2012), 2929-2939.

[20] Alper Erturk and Ghislain Delporte. 2011. Underwater thrust and power generation using flexible piezoelectric composites: an experimental investigation toward selfpowered swimmer-sensor platforms. Smart materials and Structures 20, 12 (2011), 125013.

[21] Biyi Fang, Tao Feng, Mi Zhang, and Shantanu Chakrabartty. 2015. Feasibility of B-mode diagnostic ultrasonic energy transfer and telemetry to a $\mathrm{cm} 2$ sized deep-tissue implant. In Circuits and Systems (ISCAS), 2015 IEEE International Symposium on. IEEE, 782-785.

[22] Gary D Gilbert and John C Pernicka. 1967. Improvement of underwater visibility by reduction of backscatter with a circular polarization technique. Applied Optics 6, 4 (1967), 741-746.

[23] GS1 EPCglobal Inc. 2018. EPC UHF Gen2 Air Interface Protocol. http://www. gs1.org/epcrfid/epc-rfid-uhf-air-interface-protocol/2-0-1. (2018).

[24] Raffaele Guida, Emrecan Demirors, Neil Dave, Jennie Rodowicz, and Tommaso Melodia. 2018. An Acoustically Powered Battery-less Internet of Underwater Things Platform. In 2018 Fourth Underwater Communications and Networking Conference (UComms). IEEE, 1-5.

[25] Daniel Halperin, Wenjun Hu, Anmol Sheth, and David Wetherall. 2010. Predictable 802.11 packet delivery from wireless channel measurements. In ACM SIGCOMM Computer Communication Review, Vol. 40. ACM, 159-170.

[26] EJ Hilliard Jr. 1960. Electromagnetic Radiation in Sea Water. Technical Report. Naval Underwater Ordnance Station Newport RI.

[27] Stephen Horowitz, Toshikazu Nishida, Louis Cattafesta, and Mark Sheplak. 2008. Development of a micromachined piezoelectric microphone for aeroacoustics applications. The Journal of the Acoustical Society of America 122 (01 2008), 3428-36. https://doi.org/10.1121/1.2785040

[28] Eduardo Alexander Iscar Ruland, Atulya Shree, Nicholas Goumas, and Matthew Johnson-Roberson. 2017. Low cost underwater acoustic localization. In IEEE/MTS Oceans, Vol. 30.

[29] F. M. Jaffre, P. Traykovski, M. Moulton, G. L. Lawson, and T C. Austin. 2015. Development of underwater acoustic backscatter and Doppler instruments from a small and versatile multi-frequency sonar board with software defined processing. In OCEANS 2015 - Genova. 1-6. https://doi.org/10.1109/OCEANS-Genova.2015. 7271444

[30] Sermsak Jaruwatanadilok. 2008. Underwater wireless optical communication channel modeling and performance evaluation using vector radiative transfer theory. IEEE Journal on Selected Areas in Communications 26, 9 (2008).

[31] Niels Jepsen, Eva B Thorstad, Torgeir Havn, and Martyn C Lucas. 2015. The use of external electronic tags on fish: an evaluation of tag retention and tagging effects. Animal Biotelemetry 3, 1 (2015), 49.

[32] Kiran Raj Joshi, Dinesh Bharadia, Manikanta Kotaru, and Sachin Katti. 2015. WiDeo: Fine-grained Device-free Motion Tracing using RF Backscatter.. In NSDI. 189-204.

[33] Chien-Chi Kao, Yi-Shan Lin, Geng-De Wu, and Chun-Ju Huang. 2017. A Comprehensive Study on the Internet of Underwater Things: Applications, Challenges, and Channel Models. Sensors 17, 7 (2017). https://doi.org/10.3390/s17071477

[34] Keithley Instruments, Inc. 2018. Model 2400 SourceMeter Instrument. https: //www.tek.com/datasheet/series-2400-sourcemeter-Instruments

[35] Keysight Technologies. 2019. E4990A Impedance Analyzer, $20 \mathrm{~Hz}$ to 10/20/30/50/120 MHz. https://www.keysight.com/en/pd-2405177-pn-E4990A/ impedance-analyzer-20-hz-to-10-20-30-50-120-mhz?cc=US\&lc=eng. (2019).

[36] Sri Harsha Kondapalli, Yarub Alazzawi, Marcin Malinowski, Tomasz Timek, and Shantanu Chakrabartty. 2018. Multiaccess in vivo biotelemetry using sonomicrometry and M-scan ultrasound imaging. IEEE Transactions on Biomedical Engineering 65, 1 (2018), 149-158.

[37] K. Kurokawa. 1965. Power Waves and the Scattering Matrix. IEEE Transactions on Microwave Theory and Techniques 13, 2 (March 1965), 194-202. https: //doi.org/10.1109/TMTT.1965.1125964

[38] Liu Lanbo, Zhou Shengli, and Cui Jun-Hong. 2008. Prospects and problems of wireless communication for underwater sensor networks. Wireless Communications and Mobile Computing 8, 8 (2008), 977-994.

[39] H. Li and C. Tian. 2014. Energy harvesting from low frequency applications using piezoelectric materials. Applied Physics Review, AIP 1, 4 (Nov. 2014). https://doi.org/10.1063/1.4900845

[40] H. Li, C. Tian, J. Lu, M.J. Myjak, J.J. Martinez, R.S. Brown, and Deng Z.D. 2016. An Energy Harvesting Underwater Acoustic Transmitter for Aquatic Animals. Scientific Reports 6, 33804 (2016).

[41] Ning Li, José-Fernán Martínez, Juan Manuel Meneses Chaus, and Martina Eckert. 2016. A Survey on Underwater Acoustic Sensor Network Routing Protocols. Sensors 16, 3 (2016). https://doi.org/10.3390/s16030414

[42] E. Liou, C. Kao, C. Chang, Y. Lin, and C. Huang. 2018. Internet of underwater things: Challenges and routing protocols. In 2018 IEEE International Conference on Applied System Invention (ICASI). 1171-1174. https://doi.org/10.1109/ICASI. 2018.8394494 
[43] Vincent Liu, Aaron Parks, Vamsi Talla, Shyamnath Gollakota, David Wetherall, and Joshua R Smith. 2013. Ambient backscatter: wireless communication out of thin air. ACM SIGCOMM Computer Communication Review 43, 4 (2013), 39-50.

[44] Jaime Lloret. 2013. Underwater Sensor Nodes and Networks. Sensors (Basel, Switzerland) 13 (09 2013), 11782-96. https://doi.org/10.3390/s130911782

[45] Loctite. 2019. Loctite Epoxy Marine. (2019). http://www.loctiteproducts.com/ en/products/build/epoxies/loctite epoxy_marine.html Loctite 1919324 Marine Epoxy 0.85-Fluid Ounce Syringe (1405604).

[46] Ping Lu, Xue-Song Yang, Jia-Lin Li, and Bing-Zhong Wang. 2016. Polarization reconfigurable broadband rectenna with tunable matching network for microwave power transmission. IEEE Transactions on Antennas and Propagation 64, 3 (2016), 1136-1141.

[47] Yunfei Ma, Zhihong Luo, Christoph Steiger, Giovanni Traverso, and Fadel Adib 2018. Enabling deep-tissue networking for miniature medical devices. In Proceedings of the 2018 Conference of the ACM Special Interest Group on Data Communication. ACM, 417-431.

[48] Yunfei Ma, Nicholas Selby, and Fadel Adib. 2017. Minding the Billions: Ultraw ideband Localization for Deployed RFID Tags. ACM MobiCom (2017).

[49] Warren P. Mason. 1981. Piezoelectricity, its history and applications. The journal of the Acoustical Society of America 70 (August 1981). https://doi.org/10.1121/1. 387221

[50] McMaster-Carr. 2019. Abrasion-Resistant Polyurethane Rubber Sheet. (2019). https://www.mcmaster.com/8716k61 Part No. 8716k61.

[51] Frédéric Mosca, Guillaume Matte, and Takuya Shimura. 2013. Low-frequency source for very long-range underwater communication. The Journal of the Acoustical Society of America 133, 1 (2013), EL61-EL67.

[52] NASA. 2012. Titan's Underground Ocean. https://science.nasa.gov/science-news/ science-at-nasa/2012/28jun_titanocean. (2012).

[53] P. V. Nikitin, K. V. S. Rao, S. F. Lam, V. Pillai, R. Martinez, and H. Heinrich. 2005. Power reflection coefficient analysis for complex impedances in RFID tag design. IEEE Transactions on Microwave Theory and Techniques 53, 9 (Sep. 2005), 2721-2725. https://doi.org/10.1109/TMTT.2005.854191

[54] Ali M. Niknejad. 2016. Matching Networks". http://rfic.eecs.berkeley.edu/142/ pdf/module6.pdf. (2016).

[55] PCBWay. 2019. PCBWay: China PCB Prototype \& Fabrication Manufacturer https://www.pcbway.com/. (2019).

[56] Yao Peng, Longfei Shangguan, Yue Hu, Yujie Qian, Xianshang Lin, Xiaojiang Chen, Dingyi Fang, and Kyle Jamieson. 2018. Plora: A passive long-range data network from ambient lora transmissions. In Proceedings of the 2018 Conference of the ACM Special Interest Group on Data Communication. ACM, 147-160.

[57] L. M. Pessoa, M. R. Pereira, H. M. Santos, and H. M. Salgado. 2016. Simulation and experimental evaluation of a resonant magnetic wireless power transfer system for seawater operation. In OCEANS 2016 - Shanghai. 1-5. https://doi.org/10.1109/ OCEANSAP.2016.7485704

[58] Hariharan Rahul, Farinaz Edalat, Dina Katabi, and Charles G Sodini. 2009. Frequency-aware rate adaptation and MAC protocols. In Proceedings of the 15th annual international conference on Mobile computing and networking. ACM, 193-204.

[59] Mohammad Rostami, Jeremy Gummeson, Ali Kiaghadi, and Deepak Ganesan 2018. Polymorphic radios: A new design paradigm for ultra-low power communication. In Proceedings of the 2018 Conference of the ACM Special Interest Group on Data Communication. ACM, 446-460.

[60] Yoav Y Schechner and Nir Karpel. 2003. Clear underwater vision. In Computer Vision and Pattern Recognition, 2004. CVPR 2004. Proceedings of the 2004 IEEE Computer Society Conference on, Vol. 1. IEEE, I-I.

[61] Anuj Sehgal, Catalin David, and Jãijrgen Sch ̃̃únwÃd'lder. 2011. Energy Consumption Analysis of Underwater Acoustic Sensor Networks. OCEANS'11 - MTS/IEEE Kona, Program Book. https://doi.org/10.23919/OCEANS.2011. 6107287

[62] Dongjin Seo, Jose M. Carmena, J.M. Rabaey, Elad Alon, and Michel M. Maharbiz 2013. Neural Dust: An Ultrasonic, Low Power Solution for Chronic BrainMachine Interfaces. Available at: ArXiv.org/abs/1307.2196. (07 2013).

[63] A. A. Sheikh, E. Felemban, M. Felemban, and S. B. Qaisar. 2016. Challenges and opportunities for underwater sensor networks. In 2016 12th International Conference on Innovations in Information Technology (IIT). 1-6. https://doi.org/ 10.1109/INNOVATIONS.2016.7880021

[64] Henry A Sodano, Daniel J Inman, and Gyuhae Park. 2004. A review of power harvesting from vibration using piezoelectric materials. Shock and Vibration
Digest 36, 3 (2004), 197-206.

[65] B Sai Srujana, Princy Mathews, VP Harigovindan, et al. 2015. Multi-source energy harvesting system for underwater wireless sensor networks. Procedia Computer Science 46 (2015), 1041-1048.

[66] Steminc. 2019. Piezo Ceramic Cylinder 54.1x47x40mm 17 KHz. (2019). https: //www.steminc.com/PZT/en/piezo-ceramic-cylinder-541x47x40mm-17-khz Part No. SMC5447T40111.

[67] M. Stojanovic. 2007. On the relationship between capacity and distance in an underwater acoustic communication channel. In SIGMOBILE Mobile Computing and Communications Review. ACM, 34-43.

[68] Milica Stojanovic and James Preisig. 2009. Underwater acoustic communication channels: Propagation models and statistical characterization. IEEE communications magazine 47, 1 (2009), 84-89.

[69] Thomas L Szabo. 2004. Diagnostic ultrasound imaging: inside out. Academic Press.

[70] G. W. Taylor, J. R. Burns, S. A. Kammann, W. B. Powers, and T. R. Welsh. 2001. The Energy Harvesting Eel: a small subsurface ocean/river power generator. IEEE Journal of Oceanic Engineering 26, 4 (Oct 2001), 539-547. https://doi.org/10. $1109 / 48.972090$

[71] TE Connectivity. 2015. MS5837-30BA Ultra Small Gel Filled Pressure Sensor. https://www.te.com/commerce/DocumentDelivery/DDEController?Action= srchrtrv\&DocNm=MS5837-30BA\&DocType=DS\&DocLang=English. (2015).

[72] Texas Instruments, Inc. 2012. 2-Bit Bidirectional Voltage-Level Translator with Automatic Direction Sensing. TI.

[73] Texas Instruments Inc. 2016. LMP91200 Configurable AFE for Low-Power Chemical-Sensing Applications. http://www.ti.com/lit/ds/symlink/lmp91200.pdf. (2016).

[74] Texas Instruments Inc. 2016. LP5900150-mAUltra-Low-NoiseLDO for RF and AnalogCircuits. http://www.ti.com/tool/LP5900SD-1.8EV. (2016).

[75] Texas Instruments Inc. 2019. MSP430G2553. http://www.ti.com/product/ MSP430G2553. (2019)

[76] Vo Thanh Tung, Nguyen Trong Tinh, Nguyen Hoang Yen, Anh Dang, and Dang Tuan. 2013. Evaluation of Electromechanical Coupling Factor for Piezoelectric Materials Using Finite Element Modeling. International Journal of Materials and Chemistry 2013 (12 2013), 59-63. https://doi.org/10.5923/j.ijmc.20130303.03

[77] Eva Thorstad, Audun H. Rikardsen, Ahmet Alp, and F $\tilde{A} \ddot{Y} k l a n d . ~ 2013$. The Use of Electronic Tags in Fish ResearchâASSAn Overview of Fish Telemetry Methods. Turkish Journal of Fisheries and Aquatic Sciences 13 (01 2013), 881896. https://doi.org/10.4194/1303-2712-v13_5_13

[78] Daniel M Toma, Joaquin del Rio, Montserrat Carbonell-Ventura, and Jaume Miquel Masalles. 2015. Underwater energy harvesting system based on plucked-driven piezoelectrics. In OCEANS 2015-Genova. IEEE, 1-5.

[79] Francesco Tonolini and Fadel Adib. 2018. Networking across boundaries: enabling wireless communication through the water-air interface. In Proceedings of the 2018 Conference of the ACM Special Interest Group on Data Communication. ACM, 117-131.

[80] David Tse and Pramod Viswanath. 2005. Fundamentals of wireless communication. Cambridge university press.

[81] Hao Wang, Shilian Wang, Eryang Zhang, and Luxi Lu. 2018. An Energy Balanced and Lifetime Extended Routing Protocol for Underwater Sensor Networks. Sensors 18, 5 (2018). https://doi.org/10.3390/s18051596

[82] Jue Wang, Haitham Hassanieh, Dina Katabi, and Piotr Indyk. 2012. Efficient and reliable low-power backscatter networks. In Proceedings of the ACM SIGCOMM 2012 conference on Applications, technologies, architectures, and protocols for computer communication. ACM, 61-72.

[83] WHOI. 2019. WHOI Micro-modem. (2019). https://acomms.whoi.edu/micromodem/.

[84] B. Wu, J. Liu, H. Yu, Z. Li, and Y. Chen. 2016. Underwater high-power inductive coupling energy transmission system. In OCEANS 2016 - Shanghai. 1-5. https: //doi.org/10.1109/OCEANSAP.2016.7485634

[85] Zerina Kapetanovic. 2018. WISP 5. http://wisp5.wispsensor.net/. (2018). WISP Wiki.

[86] Jingwei Zhang, Yi Huang, and Ping Cao. 2015. A microwave wireless energy harvesting system with a wideband antenna array. Transactions of the Institute of Measurement \& Control 37, 8 (2015), 961 - 969.

[87] Pengyu Zhang, Dinesh Bharadia, Kiran Joshi, and Sachin Katti. 2016. Hitchhike: Practical backscatter using commodity wifi. In ACM SENSYS. 